\title{
Rotating Neutron Stars: A Model for Pulsars*
}

\author{
M. GREWING
}

Institut für Astrophysik und Extraterrestrische Forschung der Universität Bonn

and H. HeinTZMANN

Institut für Theoretische Physik der Universität Köln

(Z. Naturforsch. 28 a, 377-382 [1973] ; received 19 October 1972)

\begin{abstract}
The main properties of neutron star matter and of neutron star models are reviewed with partic. ular emphasis on those aspects that can directly be related to pulsar observations.
\end{abstract}

\section{Introduction}

If a supernova explosion results from the gravitational collapse of a star's core into a neutron star, this neutron star is likely to begin its life with a large amount of thermal and vibrational energy. Calculations show ${ }^{1-3}$ that most of the thermal energy of a hot neutron star is rapidly dissipated by neutrinos produced in the URCA-reactions

$$
\begin{aligned}
\mathrm{n}+\mathrm{n} & \rightarrow \mathrm{n}+\mathrm{p}+\mathrm{e}+\bar{\nu}, \\
\mathrm{n}+\mathrm{p}+\mathrm{e} & \rightarrow \mathrm{n}+\mathrm{n}+v .
\end{aligned}
$$

The neutrino luminosity of a neutron star is approximately given by ${ }^{2}$

$$
L_{\nu}^{\mathrm{URCA}} \approx 2 \cdot 10^{39}(\mathrm{erg} \mathrm{sec}-1)\left(M / M_{\odot}\right) \varrho_{14}^{-1} T_{9}^{8}
$$

and if $\pi^{-}$mesons are present, cooling proceeds by means of the reactions

$$
\begin{aligned}
& \mathrm{n}+\pi^{-} \rightarrow \mathrm{n}+\mathrm{e}^{-}+\bar{v}_{\mathrm{e}}, \\
& \mathrm{n}+\mathrm{e}^{-} \rightarrow \mathrm{n}+\pi^{-}+v_{\mathrm{e}} .
\end{aligned}
$$

with a corresponding neutrino luminosity

$$
L_{\nu}^{\pi \mathrm{n}} \approx 10^{46}\left(\mathrm{erg} \mathrm{sec}^{-1}\right)\left(\begin{array}{c}
n_{\tau} \\
N
\end{array}\right)\left(\frac{M}{M_{\odot}}\right) T_{9}^{3} .
$$

Here $T_{9}$ refers to the temperature in units of $10^{9} \mathrm{~K}$; $n_{\pi}, N$ are the total pion and baryon number, respectively, and $M_{\odot}$ is the mass of the sun $\approx 2 \cdot 10^{33} \mathrm{~g}$. The heat content of a neutron star is (ignoring the latent heat in the suprafluid phase transition)

$$
u \approx N k T\left(T / T_{\mathrm{F}}\right) \approx 10^{48}(\mathrm{erg})\left(M / M_{\odot}\right) T_{9}^{2}
$$

\footnotetext{
* It is a great pleasure to dedicate this article and the preceding one "On the Origin of Cosmic Rays" to Professor KONRAD BLEULER on the occasion of his sixtieth birthday. With his broad interest both in physics and astrophysics he was among the first to realize how fruitful a cooperation between these disciplines could be. Through his own enthusiasm he has substantially promoted research across the borderlines.
}

and therefore typical cooling times are (neglecting pions)

$$
t_{\text {cooling }} \approx u / L_{\nu} \approx 4 \cdot 10^{8}(\mathrm{sec}) \varrho_{14} T_{9}^{-6}
$$

i. e. a neutron star cools below $10^{9} \mathrm{~K}$ in roughly 10 years. If however pions are included (e. g. $\left.n_{\pi} / N=10^{-2}\right)$, then

$$
t_{\text {cooling }} \approx 10^{7}(\mathrm{sec})\left(\frac{10^{2} n_{\pi}}{N}\right) \varrho_{14} T_{8}^{-4} .
$$

After the temperature has fallen below $10^{9} \mathrm{~K}$ photons provide the main cooling mechanism and neutrinos no longer play a role. Apart from a few seconds after its birth, a neutron star is therefore a cool object if one measures temperature in units of the Fermi-temperature $T_{\mathrm{F}} \approx 10^{11} \mathrm{~K}$ of the neutrons, and neutron star matter is completely degenerate if one disregards for the moment the thin surface layer. It can also be shown ${ }^{4}$ that the pulsational energy is equally quickly dissipated.

While the thermal or pulsational energy would therefore not allow one to detect a neutron star for more than a hundred years after its birth there is fortunately a better store-house for energy, namely rotation. The main question is then how this energy is transformed into observable forms of energy such as electromagnetic radiation or cosmic rays. The discovery of the unique radio-optical-X-ray and $\gamma$-ray source in the Crab nebula has provided a tentative answer. It is thought that rotation provides the basic "clock mechanism" and a strong magnetic field gives rise to the enormous radio brightness of pulsars. For completeness we repeat here the main arguments which led to the identification of pulsars with neutron stars:

1. Each of the 89 pulsars so far discovered has its own characteristic radio signature; all are, however, characterized by comparatively short pulse 
times and periods. The periods exhibit extraordinary stability and range from $33 \mathrm{msec}$ to $3.7 \mathrm{sec}$, the pulse duration being roughly $1 / 20$ of the period.

2. All periods are lengthening, $\dot{P}>0$ (at least in all pulsars where it has been measured), and this in extremely regular fashion.

3. Speed-ups have been observed in two pulsars, those in the constellation of Vela and in the Crabnebula.

4. In case of the pulsar in the Crab-nebula, the pulsar is associated with the remnant of a supernova which was seen by Chinese and Japanese astronomers for 23 days in 1054 A.D. The "age" of the pulsar and the typical slow-down time $t=P / \dot{P}$ nearly coincide in this case. The second fastest pulsar in the Vela constellation is equally associated with a remnant of a supernova which - from theoretical consideration - is estimated to be some $10^{4}$ years old, again in agreement with the corresponding $t=P / \dot{P}=2.2 \cdot 10^{4}$ years.

5 . The loss of energy per time due to the slowdown, $\dot{E}=I \Omega \dot{\Omega}$, is in agreement with that needed to keep the Crab nebula ablaze: the pulsar at its centre makes the Crab shine. Most of the energy output of the nebula is in form of soft X-rays. It is however known that the spectrum reaches up to $\gamma$ ray-energies. To produce these high-energy photons, ultrarelativistic electrons of energy $\approx 10^{14} \mathrm{eV}$ have to be continuously supplied into the magnetic field of the nebula*. It is tempting therefore to assume that the pulsar is also a source of high energy cosmic rays.

All these findings find a natural explanation in the rotating neutron star model. Since ordinary stars rotate and posses magnetic fields, one expects that neutron stars will also.

If one further assumes, as a first approximation, that magnetic flux (a good approximation) and angular momentum (probably not so good an approximation) are conserved in the collapse of a star of $M>1.4 M_{\odot}$ with $\Omega \approx \Omega_{\odot}=3 \cdot 10^{-6} \mathrm{sec}^{-1}$ and a magetic field $B_{0}$, then the resulting neutron star would have its angular frequency and magnetic field

* The relations between critical frequency $\nu_{\mathrm{c}}$, lifetime $\tau$, and electron energy $\varepsilon$ in a magnetic field $B$ are

$$
\begin{aligned}
\tau & =8,4 \cdot 10^{-2} B_{-3}^{-2} \varepsilon_{14}^{-2} \text { (years), } \\
v_{\mathrm{c}} & =\frac{3}{4 \pi} \frac{e B \perp}{m c} \gamma^{2}=1,6 \cdot 10^{20} \varepsilon_{14} B_{-3} \text { (Hertz), }
\end{aligned}
$$

$B$ in Gauss, $\varepsilon$ in $\mathrm{eV}$. enlarged by a factor $\approx\left(R_{\odot} / R\right)^{2} \approx 10^{10}$. The magnetic field need not, in general, point along the axis of rotation, and could thus give rise to a directivity. The star would act as a big unipolarinductor, ripping charged particles from its surface, accelerating them to relativistic energies. While therefore most findings we have mentioned above do find a tempting explanation in the rotating neutron star model, the very process behind the phenomenon through which pulsars are observed remains unclear. With this general picture in mind we shall describe here briefly the main properties of neutron star matter and neutron star models and in more detail those aspects which have been elaborated upon in Bonn ${ }^{5,6}$ and $\mathrm{Köln}{ }^{7,8}$.

\section{The Equation of State of Neutron Star Matter}

The equation of state and the static properties of neutron star matter are investigated in the region below $4 \cdot 10^{14} \mathrm{~g} / \mathrm{cm}^{3}$ by means of different improved techniques for handling the nuclear many-body problem. While most observations are probably related to the physics of the outermost crust and magnetosphere of the pulsar, neutron star models put useful constraints to explain the energy output, pulse finestructure and most drastically the occasional speedups of the pulse-rate. Moreover, an understanding of the physical properties of neutron-star-matter helps to find out how ultimately observable amounts of energy can be stored in rotating neutron stars over astronomical time-scales.

A magnetic field of $10^{13}$ Gauss corresponds to an equivalent mass density of

$$
\varrho \approx B^{2} / 8 \pi c^{2} \approx 5 \cdot 10^{3} \mathrm{~g} / \mathrm{cm}^{3} .
$$

The outermost layer of a neutron star is therefore dominated by the magnetic field, a fact which considerably complicates a study of the equation of state and its physical properties. In the present study, magnetic fields will be disregarded as we are mainly interested in the properties of extremely dense neutron star matter where its influence can be safely neglected. (Its interaction energy with electrons is of order $\mu_{\mathrm{e}} B \approx 10^{5} \mathrm{eV}$ and some $10^{2} \mathrm{eV}$ with nuclei, which is negligible for densities beyond $10^{6} \mathrm{~g} / \mathrm{cm}^{3}$.)

Beneath this layer of essentially unknown composition and structure there is a solid crust which extends up to some $10^{14} \mathrm{~g} / \mathrm{cm}^{3}$. 
The static properties (equation of state, composition) for this crustal regime of neutron stars have been derived from a concept to handle the nuclear binding, which could be adjusted in order to yield at the same time the experimental masses of ordinary nuclei ${ }^{8}$.

The basic results are:

1. At densities from $\varrho_{1} \approx 3 \cdot 10^{6} \mathrm{~g} / \mathrm{cm}^{3}$ to $\varrho_{2}=4.69$ $\cdot 10^{11} \mathrm{~g} / \mathrm{cm}^{3}$ the matter is a solid and consists of more and more neutron-rich nuclei embedded in a degenerate relativistic electron gas. At the density $\varrho_{2}$ (where the neutrons drip out of the nuclei) the electron chemical potential is $26.0 \mathrm{MeV}$ in this calculation, thus being exactly the same as that of BAYM et al. ${ }^{9}$ at their $n$-drip-density $\varrho_{2}=4.3 \cdot 10^{11} \mathrm{~g} / \mathrm{cm}^{3}$.

2. Above $\varrho_{2}$ the nuclei start to evaporate free neutrons; thus the matter is composed of extremely neutron-rich nuclei embedded in a strongly interacting neutron gas and the electron gas (a proton gas has not been found to exist simultaneously with the nuclei).

3. At a density $\varrho_{3} \approx 10^{14} \mathrm{~g} / \mathrm{cm}^{3}$ the nuclei disintegrate and the matter transforms by a phase transition of first order to a homogeneous gas of neutrons, protons and electrons. The jump $\Delta \varrho / \varrho$ of the matter density at the transition point is $\approx 6 \%$ and the transition ("evaporation") heat $\Delta u / u \approx 5.8 \%$. In Tables 1 and 2 we have summarized the properties of neutron star matter which follow from calculations by BAYM et al. ${ }^{9}$ and Reference ${ }^{8}$.

Table 1. Equation of state of neutron star matter. $\varrho$ is the density, $P$ the pressure, $Z$ and $A$ charge and atomic weight of the nuclei, $\mu_{\mathrm{e}}$ the electron chemical potential, $T_{\mathrm{m}}$ the melting temperature of the lattice $T_{\mathrm{m}} \approx 10^{7} \varrho_{6}^{1 / 3}(\mathrm{Z} / 8)^{5 / 3}$ see Reference ${ }^{15}$.

\begin{tabular}{rlllll}
\hline$\varrho$ & $P$ & $A$ & $\begin{array}{l}\mu_{\mathrm{e}} \\
(\mathrm{MeV})\end{array}$ & $\begin{array}{l}T_{\mathrm{m}} \\
\left(10^{8}{ }^{\circ} \mathrm{K}\right)\end{array}$ \\
& $\begin{array}{l}\left.\mathrm{gcm}^{-3}\right) \\
(\mathrm{dynes})\end{array}$ & & & & \\
\hline $10^{4}$ & $9.7 \cdot 10^{18}$ & 26 & 56 & & \\
$1.6 \cdot 10^{5}$ & $1.1 \cdot 10^{21}$ & 26 & 56 & & \\
$10^{6}$ & $2.3 \cdot 10^{22}$ & 26 & 56 & & \\
$8.3 \cdot 10^{6}$ & $5.3 \cdot 10^{23}$ & 28 & 56 & 0.95 & 1.6 \\
$1.3 \cdot 10^{8}$ & $2.6 \cdot 10^{25}$ & 28 & 56 & 2.1 & 2 \\
$10^{9}$ & $4.1 \cdot 10^{26}$ & 28 & 64 & 3.8 & 6 \\
$10^{10}$ & $7.7 \cdot 10^{27}$ & 32 & 82 & 8.2 & 20 \\
$10^{11}$ & $1.5 \cdot 10^{28}$ & 28 & 78 & 17 & 37 \\
$3.3 \cdot 10^{11}$ & $5.8 \cdot 10^{29}$ & 38 & 120 & 24 & 70 \\
\hline
\end{tabular}

Eventually the nuclei disappear, and the matter becomes an isotropic and homogeneous mixture of neutrons, protons, electrons. Both neutrons and pro- tons might be superfluid, the protons thus giving rise to proton superconductivity. For the determination of the equation of state, superfluidity is of minor importance since according to all calculations performed so far their condensation energy is small save around $10^{12} \mathrm{~g} / \mathrm{cm}^{3}$ where it could give rise to a major first order phase transition. In the past, a wide variety of calculations related to neutron-starmatter or pure neutron-matter have been performed, and the results that various authors have obtained differ greatly from each other. Both the minimum and maximum allowed mass for stable, bound neutron-stars depend in a sensitive way on the properties of neutron-star-matter. This makes it worthwhile to try to eliminate as much uncertainty as possible in the region up to $\varrho \approx 10^{15} \mathrm{~g} / \mathrm{cm}^{3}$, the limit at which the validity of non-relativistic many-body theory ceases, and to study model-extensions to still higher densities at which the maximum mass of the neutronstar sequence is reached. Neutrons, which are the dominant component of such neutron-star-matter, are responsible for most of the pressure and compressibility, and a study of the properties of the pure neutron gas is of prime importance.

However all calculations - especially beyond nuclear densities - are plagued by two difficulties

a) an incomplete knowledge of the nucleon-nucleoninteraction and

b) the lack of reliable methods for evaluating the interaction energy of a system of strongly interacting particles.

Furthermore, the uncertainties associated with a) and b) are not easy to isolate ${ }^{5-7}$.

Recently Buchler and Ingber ${ }^{10}$ and Siemens and PANDharipande ${ }^{11}$ have applied lowest-order Brueckner-theory to different interaction-potentials, and Clark and collaborators have used the method of correlated basis functions both for hard and softcore potentials ${ }^{12}$. Yet other methods have been developed to treat the nuclear many-body problem ${ }^{5,6}$ and results of these earlier investigations have been summarized in ${ }^{7}$.

In nuclear physics the energy per particle $E / N$ is conveniently expressed by means of the Fermi-wave number $k_{\mathrm{F}}\left(\mathrm{fm}^{-1}\right)$ which is related to the particle density $n=3 \pi^{2} k_{\mathrm{F}}{ }^{3}$. In the low-density gas approach the interaction energy is expanded in a series of $k_{\mathrm{F}}$, the kinetic energy being $E_{\mathrm{kin}} / N=a k_{\mathrm{F}}^{2}$ with $a=$ 14.44 $\mathrm{MeV} \mathrm{fm}^{2}$. The lowest-order contributions to 
the potential energy are $E_{\mathrm{pot}} / N=b k_{\mathrm{F}}{ }^{3}+c k_{\mathrm{F}}{ }^{5}+\ldots$ the coefficients being independent of $k_{\mathrm{F}}$. In principle it would therefore suffice to know $E / N$ over a comparatively small density interval to high accuracy to determine the various coefficients. These would allow then to extrapolate $E / N$ to high densities. For all cases studied we find that $E / N$ can indeed be fitted over the whole density range of interest by

$$
W=E / N=a k_{\mathrm{F}}^{2}+b k_{\mathrm{F}}^{3}+c k_{\mathrm{F}}^{5}
$$

however it is sometimes convenient to consider $a$ as a free parameter to include possible electronproton contributions, or to allow for a $k_{\mathrm{F}}{ }^{7}$-term to describe the very high density regime. In the low density regime where a Coulomb-lattice is present, analytic fits are more involved.

Once we have $W$ as a function of $k_{\mathrm{F}}$ or equivalently $n$, we find the energy density $\varepsilon:=n W$, the chemical potential $\mu:=\mathrm{d}(n W) / \mathrm{d} n$, the pressure $P$ : $P=n \mu-\varepsilon$, the compressibility $\varkappa: 1 / \varkappa=n(\mathrm{~d} P / \mathrm{d} n)$ and the adiabatic index $\Gamma: T=1 / \varkappa P$ by simple algebraic manipulations. Results of such calculations are summarized in Table 2.

\section{Neutron Star Parameters}

From the equation of state one obtains the parameters of neutron star models by integration of the equations of hydrostatic equilibrium. In neutron stars, relativistic effects are strong, and a fully relativistic theory of gravitation has to be used. It turns out that in both rival theories - the original Einstein theory of general relativity and the scalartensor theory by Jordan-Brans-Dicke which are most discussed today - neutron star models are very similar ${ }^{13,14}$, and we shall confine our attention here to Einsteinian models. The equation of hydrostatic equilibrium was derived by Tolman, Oppenheimer and Volkov (T.O.V. equation) and reads

$$
\begin{aligned}
\mathrm{d} M / \mathrm{d} r & =4 \pi r^{2} \varrho \\
-\mathrm{d} P / \mathrm{d} r & =G \begin{array}{c}
\left(\varrho+P / c^{2}\right)\left(M+4 \pi r^{3} P / c^{2}\right) \\
r^{2}\left(1-2 G M / r c^{2}\right)
\end{array} .
\end{aligned}
$$

Here $\varrho$ is the rest mass plus potential and kinetic energy density $\varrho=m n+\varepsilon / c^{2}$. Newton's equations are obtained by letting $c \rightarrow \infty$. The radius $R$ of the star is defined as usual by $P(R)=0$, the gravitating mass of the star is $M$, and the total number of baryons

$$
N=\int_{0}^{R} \frac{4 \pi r^{2} n(r) \mathrm{d} r}{\left(1-2 G M(r) / r^{2} c\right)^{1 / 2}} .
$$

The difference between the mass of $N$ dispersed baryons (He-atoms for example, if the neutron star is formed from a star which has processed through He-burning) and $M c^{2}$ is the mass defect

$$
\Delta=\left(N m_{\mathrm{He}}-M\right) / N m_{\mathrm{He}} .
$$

In Tables 3 and 4 we have listed white dwarf- and neutron-stellar paramaters which have been obtained by integrating the equations of state described above. From Table 4 we see that $\Delta$ exceeds $10 \%$ which means that heavy neutron stars can convert a considerable fraction of their rest mass into energy compared to $\approx 1 \%$ in the fusion of $56 \mathrm{H} \rightarrow \mathrm{Fe}_{56}$. The birth of a neutron star will therefore be a spectacular event since roughly $0.1 M_{\odot} c^{2} \approx 10^{53}$ ergs will be set free!

\begin{tabular}{|c|c|c|c|c|c|c|c|c|c|c|}
\hline$\varrho_{\left(\mathrm{gcm}^{-3}\right)}$ & $\begin{array}{l}P \\
\left(\mathrm{MeV} \mathrm{fm}^{-3}\right)\end{array}$ & $Z$ & $A$ & $\begin{array}{l}\mu_{\mathrm{e}} \\
(\mathrm{MeV})\end{array}$ & $\begin{array}{l}\mu_{\mathrm{n}} \\
(\mathrm{MeV})\end{array}$ & $T_{\mathrm{m}}$ & $\begin{array}{l}X \\
(\%)\end{array}$ & $\begin{array}{l}T_{\mathrm{c}, \mathrm{n}} \\
\left(10^{9} \mathrm{~K}\right)\end{array}$ & $H_{\mathrm{c}, 15}$ & $\begin{array}{l}T_{\mathrm{c}, \mathrm{p}} \\
\left(10^{9} \mathrm{~K}\right)\end{array}$ \\
\hline $4.6 \cdot 10^{11}$ & $0.42 \cdot 10^{-3}$ & 36 & 111 & 24.9 & 0.28 & 5 & 24.7 & 2.6 & - & - \\
\hline $1.2 \cdot 10^{12}$ & $0.72 \cdot 10^{-3}$ & 37 & 118 & 27.2 & 0.93 & 13 & 12.3 & 6 & - & - \\
\hline $3 \cdot 10^{12}$ & $1.84 \cdot 10^{-3}$ & 38 & 129 & 30.4 & 1.85 & 20 & 6.8 & 9,8 & - & - \\
\hline $10^{13}$ & $8.78 \cdot 10^{-3}$ & 40 & 154 & 37.7 & 3.71 & 30 & 3.8 & 7 & - & - \\
\hline $4 \cdot 10^{13}$ & $5.14 \cdot 10^{-2}$ & 39 & 211 & 52.5 & 6.88 & 50 & 2.7 & 5 & 0.3 & 1 \\
\hline $1.1 \cdot 10^{14}$ & 0.275 & 25 & 349 & 76.4 & 11.8 & 32 & 2.9 & 2 & 2.0 & 3 \\
\hline $3 \cdot 10^{14}$ & 3.15 & - & - & 128 & 33.2 & - & 6.0 & $?$ & 4.9 & 5 \\
\hline $4.7 \cdot 10^{14}$ & 9.3 & - & - & 152 & 59.3 & - & 8.0 & $?$ & 2.8 & 2.8 \\
\hline $9 \cdot 10^{14}$ & 49.9 & - & - & 207 & 158 & - & 11.6 & $?$ & - & - \\
\hline $2 \cdot 10^{15}$ & 256 & - & - & 315 & 399 & - & 21.2 & - & - & - \\
\hline
\end{tabular}

Table 2. $X$ : percentage of charged particles, $\mu_{\mathrm{n}}$ : chemical potential of neutrons, $T_{\mathrm{m}}$ : melting temperature of lattice, $T_{\mathrm{c}, \mathrm{n}}:$ critical temperature of ${ }^{1} \mathrm{~S}_{0} \mathrm{n}$-superfluidity, $H_{\mathrm{c}, \mathrm{n}}$ : critical magnetic field in units $10^{15} \mathrm{Gauss}, P$ is the pressure in $\left(\mathrm{MeV}^{1} \mathrm{fm}^{-3}\right) \triangleq$ $6.25 \cdot 10^{32}\left(\right.$ dynes $\left.\mathrm{cm}^{-2}\right), T_{\mathrm{c}, \mathrm{p}}$ : critical temperature for proton ${ }^{1} \mathrm{~S}_{0}$ superconductivity. The different quantities are from HEINTZManN et al. ${ }^{8}$, and from H. Yang, J. W. Clark, Lett. Nouvo Cim. 4, 969 [1972]. 
Table 3. $\varrho_{\mathrm{c}}$ : central density, $R$ : radius, $E_{\mathrm{bind} / N}$ : binding energy per particle with respect to dispersed Helium ${ }^{*}, N$ : total number of particles, $I:$ moment of inertia. ${ }^{*} E_{\text {bind }}(\mathrm{He}$ lium) $=-7.15 \mathrm{MeV} /$ particle.

\begin{tabular}{llllll}
\hline $\begin{array}{l}Q \mathrm{c} \\
\left(\mathrm{gcm}^{-3}\right)\end{array}$ & $\begin{array}{l}M \\
(M \odot)\end{array}$ & $\begin{array}{l}R \\
(\mathrm{~km})\end{array}$ & $\begin{array}{l}E_{\mathrm{bind} / N} \\
(\mathrm{MeV})\end{array}$ & $\begin{array}{l}N \\
\left(10^{57}\right)\end{array}$ & $\begin{array}{l}I \\
\left(10^{50} \mathrm{gcm}^{2}\right)\end{array}$ \\
\hline $1.66 \cdot 10^{6}$ & 0.40 & 9900 & -2.31 & 0.48 & 1.5 \\
$8.5 \cdot 10^{7}$ & 0.92 & 4070 & -2.42 & 1.10 & 0.46 \\
$5.0 \cdot 10^{8}$ & 1.03 & 2760 & -2.46 & 1.2 & 0.20 \\
$2.6 \cdot 10^{9}$ & 1.00 & 1860 & -2.44 & 1.2 & 0.07 \\
\hline
\end{tabular}

As we have mentioned in the introduction, rotation is the main storehouse for energy. The rest of our considerations will therefore be devoted to rotational properties of neutron stars. In Newtonian theory the moment of inertia for a rigidly rotating sphere is given by

$$
I_{\text {Newton }}=(8 \pi / 3) \int \varrho r^{4} \mathrm{~d} r .
$$

In Einstein's theory it is very difficult to describe fully relativistic objects rotating relativistically.

However for slow rotation $\Omega \ll\left(G M / R^{3}\right)^{1 / 2}$ one can linearize Einstein's field equations with respect to $\Omega\left(R^{3} / G M\right)^{1 / 2}$, and one finds for the moment of inertia $^{8}$

$$
I_{\text {Einstein }}=\frac{8 \pi}{3} \int_{0}^{R} \mathrm{~d} r \frac{r^{4}\left(\varrho+P / c^{2}\right)}{\left(1-2 G M / r c^{2}\right)^{1 / 2}} \frac{\bar{\omega}(\bar{v})}{\Omega} e^{-v / 2}
$$

where

$$
e^{v / 2}=\left\{\exp \int_{0}^{p=p(r)} \mathrm{d} p / p+\varrho c^{2}\right\}\left(1-2 G M / r c^{2}\right)^{1 / 2},
$$

and $\bar{\omega}(r)$ describes the dragging of inertial frames. It obeys a differential equation which has to be solved along with the TOV-equation:

$$
\begin{aligned}
& \left(\left(1-2 G M / r c^{2}\right)^{1 / 2} e^{-v / 2} r^{4} \bar{\omega}^{\prime}\right)^{\prime} \\
& \quad=\frac{16 \pi G r^{4}}{c^{2}} \frac{e^{-\nu / 2}}{\left(1-2 G M / r c^{2}\right)^{1 / 2}}\left(\varrho+P / c^{2}\right) \bar{\omega}
\end{aligned}
$$

with the boundary conditions:

$$
\bar{\omega}(\infty)=\Omega, \quad \bar{\omega}^{\prime} \underset{r \rightarrow 0}{\sim A} r .
$$

The moment of inertia $I$ for our equation of state is given in Table 4 . The general relativistic effects on the moment of inertia become important only for very massive stars, and for our equation of state the ratio between $I_{\mathrm{E}}$ and $I_{\mathrm{N}}$ is $I_{\mathrm{E}} / I_{\mathrm{N}} \approx 2$ for them; the relativistic moment of inertia is therefore considerably larger than would follow from Newtonian theory.

\section{Transport Processes}

If the rotation of neutron stars is related to the pulsar pulse-periods, then observations tell us that neutron stars are being slowed down, and must loose angular momentum to the surrounding interstellar gas or nebula. Angular momentum has therefore to be transported both away from the surface and down to the core of the neutron star. In this section we will discuss qualitatively how the transport of angular momentum might be achieved microscopically.

In Fig. 1 we have depicted the cross section of a typical neutron star. Beneath a layer which is a few

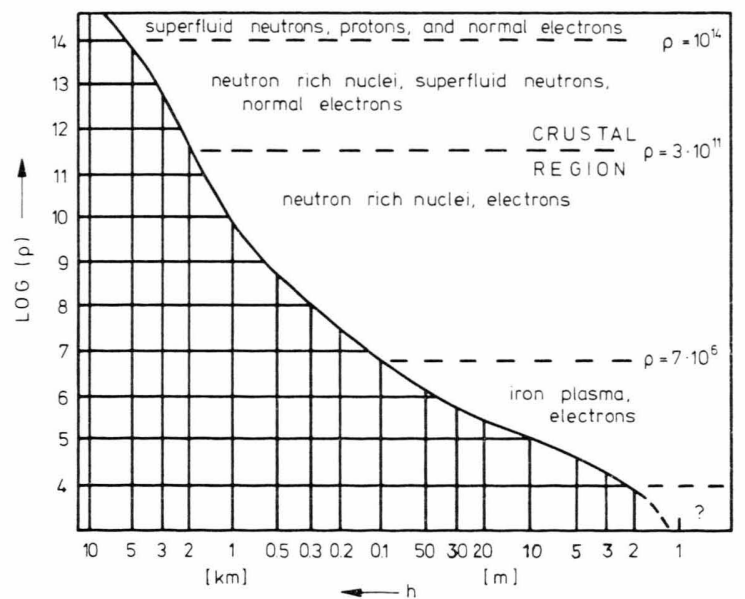

\begin{tabular}{|c|c|c|c|c|c|c|}
\hline $\begin{array}{l}\varrho_{c} \\
\left(10^{14} \mathrm{gcm}^{3}\right)\end{array}$ & $\begin{array}{l}M \\
(M \odot)\end{array}$ & $\begin{array}{l}R \\
\mathrm{~km}\end{array}$ & $\Delta$ & ${\stackrel{I}{\left(10^{44} \mathrm{gcm}^{2}\right)}}$ & $\begin{array}{l}N \\
\left(10^{57}\right)\end{array}$ & $\sigma$ \\
\hline 1.7 & 0.09 & 650 & $3.5 \cdot 10^{-3}$ & 26.8 & 0.11 & $4 \cdot 10^{-3}$ \\
\hline 2.7 & 0.17 & 17.5 & $-3.4 \cdot 10^{-3}$ & 0.8 & 0.20 & $3 \cdot 10^{-2}$ \\
\hline 6.3 & 0.66 & 11.8 & $-4.2 \cdot 10^{-2}$ & 5 & 0.83 & 0.162 \\
\hline 10 & 1.11 & 11.3 & $-7.7 \cdot 10^{-2}$ & 11 & 1.4 & 0.29 \\
\hline 20 & 1.55 & 10.3 & -0.118 & 16 & 2.1 & 0.435 \\
\hline 30 & 1.63 & 9.7 & -0.125 & 15 & 2.2 & 0.44 \\
\hline
\end{tabular}

Fig. 1. Cross section through a medium heavy neutron star, $\varrho_{\mathrm{c}}=5 \cdot 10^{14} \mathrm{gcm}^{-3}, R=11.9 \mathrm{~km}, \mathrm{M}=0.26 \mathrm{M}, T_{\mathrm{c}}=$ $10^{8} \mathrm{~K}$. The distance $h$ is measured from the surface of the star.

Table 4. $\Delta$ : Massdefect, $\left.\sigma:(2 G M) / c^{2} R\right)=R_{\mathrm{S}} / R, \quad R_{\mathrm{S}}=2,9 \mathrm{~km} \cdot M / M \odot$. 
meters thick and the structure of which is dominated by the strong magnetic field, there is a plasma of iron nuclei and electrons. From Table 1 we see that around $10^{8} \mathrm{~g} / \mathrm{cm}^{3}$ the plasma will solidify if the internal temperature is of the order of $5 \cdot 10^{8} \mathrm{~K}$, a temperature which a neutron star will have reached after a thousand years of cooling. Beginning some hundred meters (or only a few meters in heavier neutron stars) below the surface, neutron star matter will contain a crystal lattice that can support transverse shear. The shear modulus of a Coulomb lattice has been estimated to be ${ }^{16,17}$

$$
\mu \approx(Z e)^{2} n_{z}^{4 / 3}, \quad n_{z}=\text { number density of nuclei, }
$$

and this is more than sufficient to transport the torque down the star to where the superfluid neutron and proton component starts. However the electrons which must be present to establish equilibrium between protons and neutrons

$$
\mu_{\mathrm{n}}=\mu_{\mathrm{e}}+\mu_{\mathrm{p}}
$$

are still normal. Therefore if there was no magnetic field, electron-electron scattering would lead to a viscosity ${ }^{4}$

$$
\begin{aligned}
\eta & =\frac{1}{3} \varrho \tau_{\mathrm{el}} v_{\mathrm{s}}{ }^{2} \\
\tau_{\mathrm{el} \mathrm{el}} & \approx \alpha^{-3 / 2} \frac{\hbar}{\mu_{\mathrm{e}}}\left(\frac{T_{\mathrm{el}}}{T}\right)^{2} \approx 3 \cdot 10^{-14}\left(\frac{\mu_{\mathrm{e}}}{\mathrm{MeV}}\right) T_{8}^{-2} \mathrm{sec} \\
v_{\mathrm{s}} & =\text { velocity of sound. }
\end{aligned}
$$

In the stationary case the fluid would rotate differen. tially with a rate $(\Delta \dot{n})=-(\dot{\Omega} / 2 \pi)\left(R^{2} \varrho / 10 \eta\right)$.

For the $\dot{\Omega}$ of the Crab nebula pulsar this would imply that the inner portion would overtake the crust

1 H. Y. Chiu and E. E. Salpeter, Phys. Rev. Lett. 12, 413 [1964].

2 J. N. Bahcall and R. A. Wolf, Phys. Rev. 140, B 1452 [1965].

3 A. Finzi and R. A. Wolf, Astrophys. J. 153, 835 [1968].

4 H. Heintzmann and J. Nitsch, Astron. Atroph. 21, 291 [1972].

5 K. Hohlinde, K. Erkelenz, and R. Alzetta, Nucl. Phys. A. 194, 161 [1972].

${ }^{6}$ K. Bleuler, K. Erkelenz, K. Hohlinde, and R. MachLEIDT, Nucl. Phys. A, in press.

7 J. W. Clark, H. Heintzmann, W. Hillebrandt, and M. GREwing, Astrophys. Lett. 10, 21 [1971].

8 H. Heintzmann, W. Hillebrandt, M. F. El Eid, and E. Hilf, Astron. Astroph., in press.

9 G. Baym, Ch. Pethnick, and P. Sutherland, Astrophys. J. 170, 299 [1971], roughly $100 T_{8}^{2}$ times per year. The protons are strongly coupled to the electrons because otherwise extremely large magnetic fields would have to be built up, which is impossible because of the good electrical conductivity ${ }^{18}$. However the neutrons are only coupled weakly to the electrons through the normal cores of their vortices ${ }^{19}$, and the relaxation times can reach days or more so that the neutrons will always be faster than the charged component by

$$
\Delta \Omega \approx-\dot{\Omega} \tau_{\mathrm{eln}}\left(I_{\mathrm{n}} / I_{\mathrm{c}}\right)
$$

where $\tau_{\mathrm{eln}}$ is the relaxation time between charged particles and neutrons, $I_{\mathrm{n}}, I_{\mathrm{c}}$ the moments of inertia of the superfluid (neutral) and charged component respectively.

In the presence of a strong magnetic field the torque is transported by means of magnetic stresses. One finds that the torque

$$
M_{\mathrm{Z}}=\left(R^{3} / 4 \pi\right) \int \mathrm{d} \Omega B_{r} B_{\varphi} \sin ^{2} \vartheta
$$

implies a torodial magnetic field

$$
B_{\varphi}=3 I \dot{\Omega} / R^{3} B_{r},
$$

and even in the case of the Crab nebula pulsar it is only of the order of $3 \cdot 10^{6}$ (Gauss) $R_{6}{ }^{-3} B_{12}{ }^{-1}$. These considerations also apply to the thin surface layer, and the corotating part of the magnetosphere. The remaining and difficult problem is therefore to find out how the torque is imparted from the corotating magnetosphere to the plasma further away, and finally to the interstellar gas or nebula. Qualitative considerations can be found in an interesting paper by JULIAN and GOLDREICH ${ }^{20}$.

10 J. R. Buchler and L. Ingber, Nucl. Phys. A 170, 1 [1971].

11 P. J. Siemens and V. R. Pandharipande, Nucl. Phys. A 173, 561 [1971].

12 M. Miller, C. W. Woo, J. W. Clark, and W. Ter Low, Nucl. Phys. A 184, 1 [1972].

13 A. Salmona, Phys. Rev. 154, 1218 [1967].

14 W. Hillebrandt and H. Heintzmann, to be published.

15 M. A. Ruderman, J. Physique 30, C 3, 152 [1969].

16 C. B. Clark, Phys. Rev. 109, 1133 [1958].

17 M. A. Ruderman, Nature London 223, 597 [1969].

18 M. Grewing and H. Heintzmann, Z. Physik 250, 254 [1972].

19 G. Baym, C. Pethick, D. Pines, and P. Feibelman, Nature London 224, 673 [1969].

20 P. Goldreich and W. H. Julian, Astrophys. J. 157, 869 [1969]. 